\title{
Deconfinement, monopoles and new phenomena in heavy ion collisions
}

\section{Edward Shuryak}

(Shifmania, May 2009)

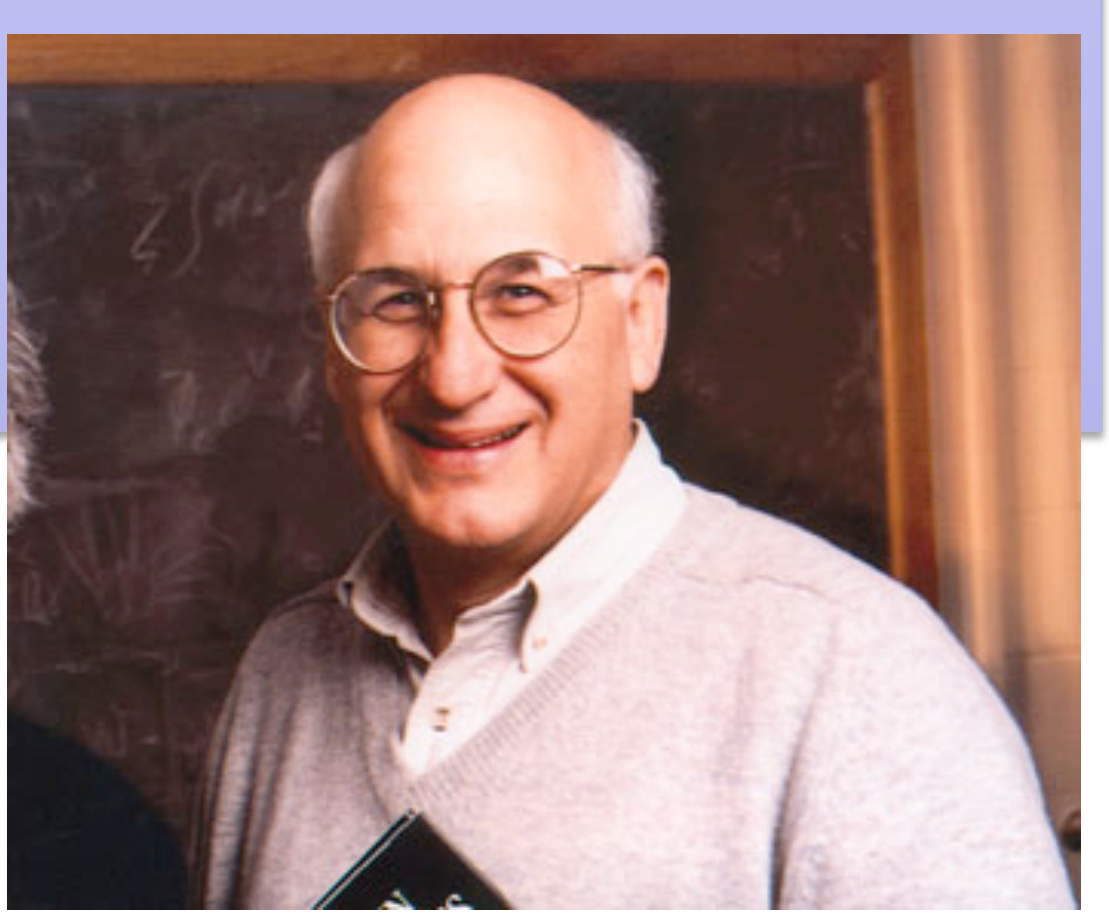

"... a very long lived excited state" (compressed, by me, version of Voloshin introduction) 


\section{Nor-Amberd, 1980's}

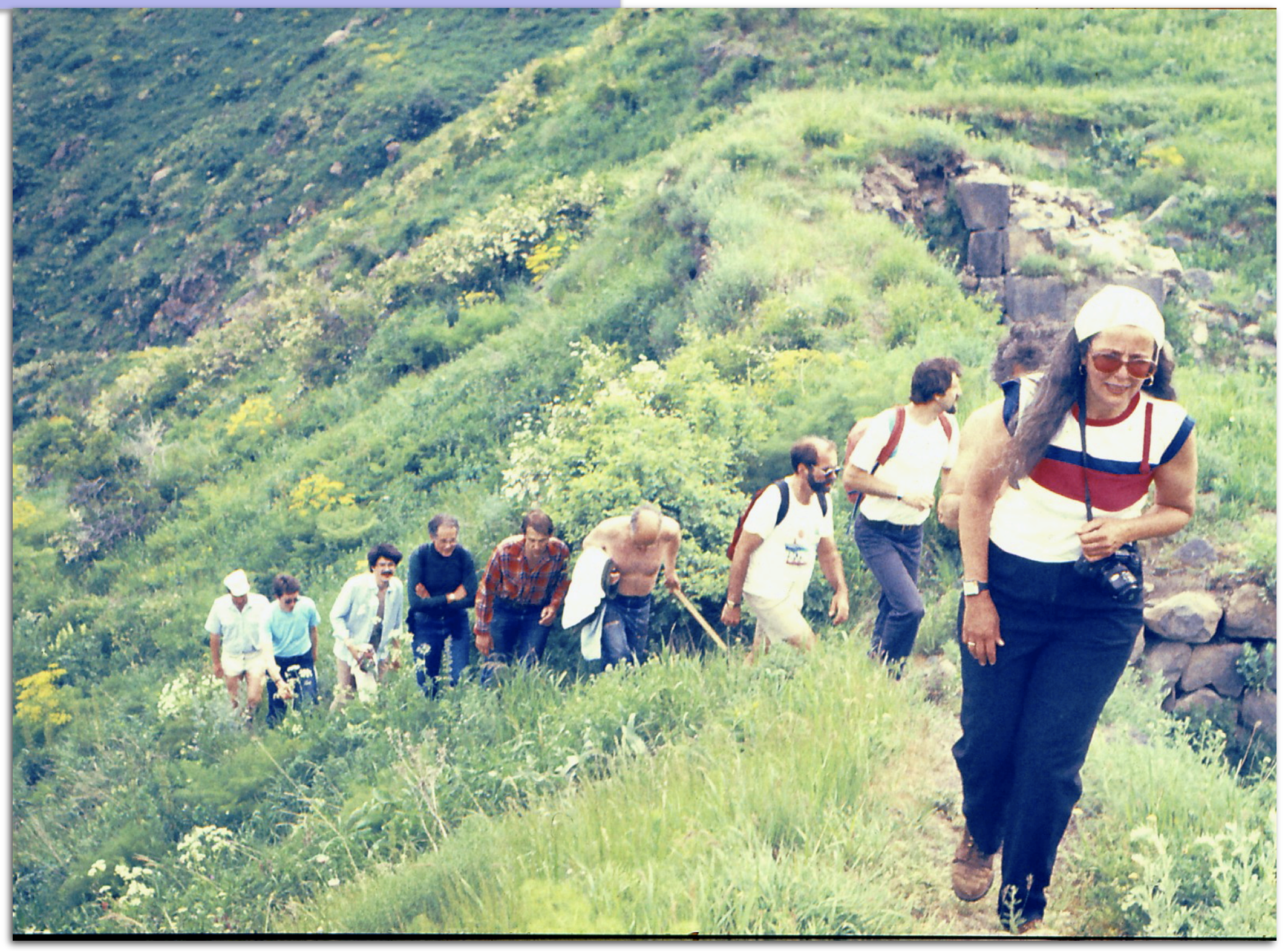




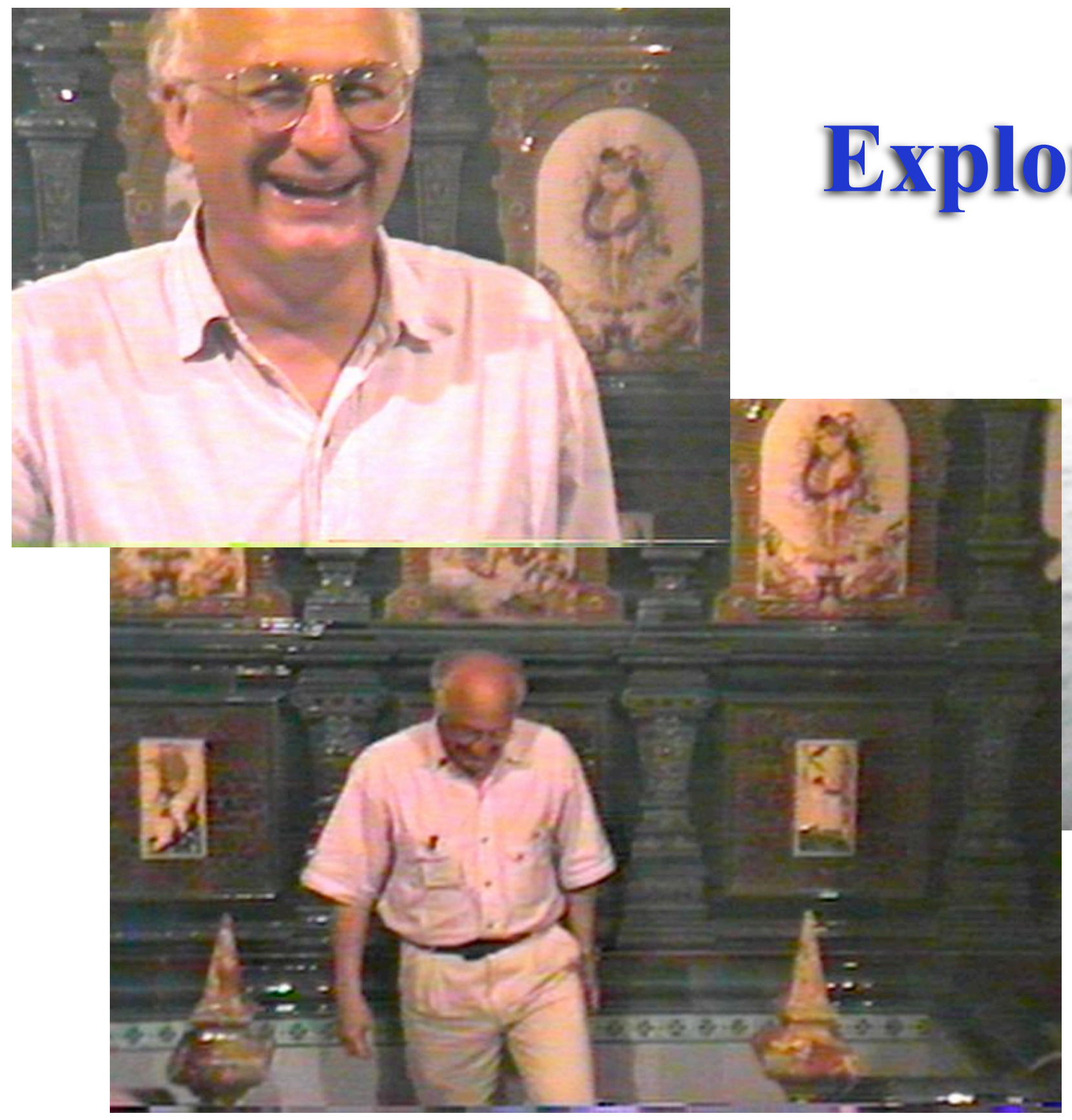

\section{Exploring bathing}

Como, 1990's

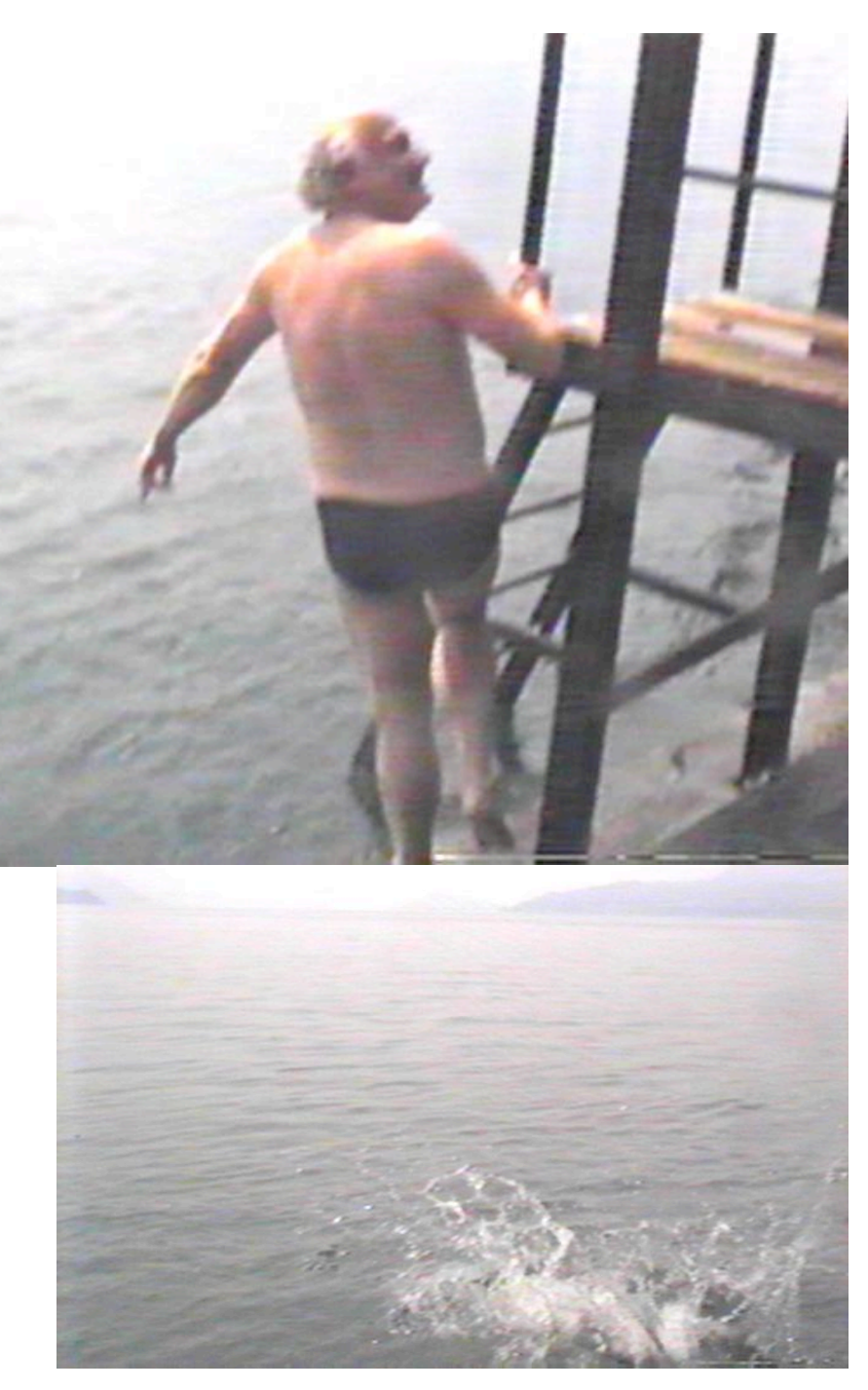




\section{The emerging theory of SQGP}

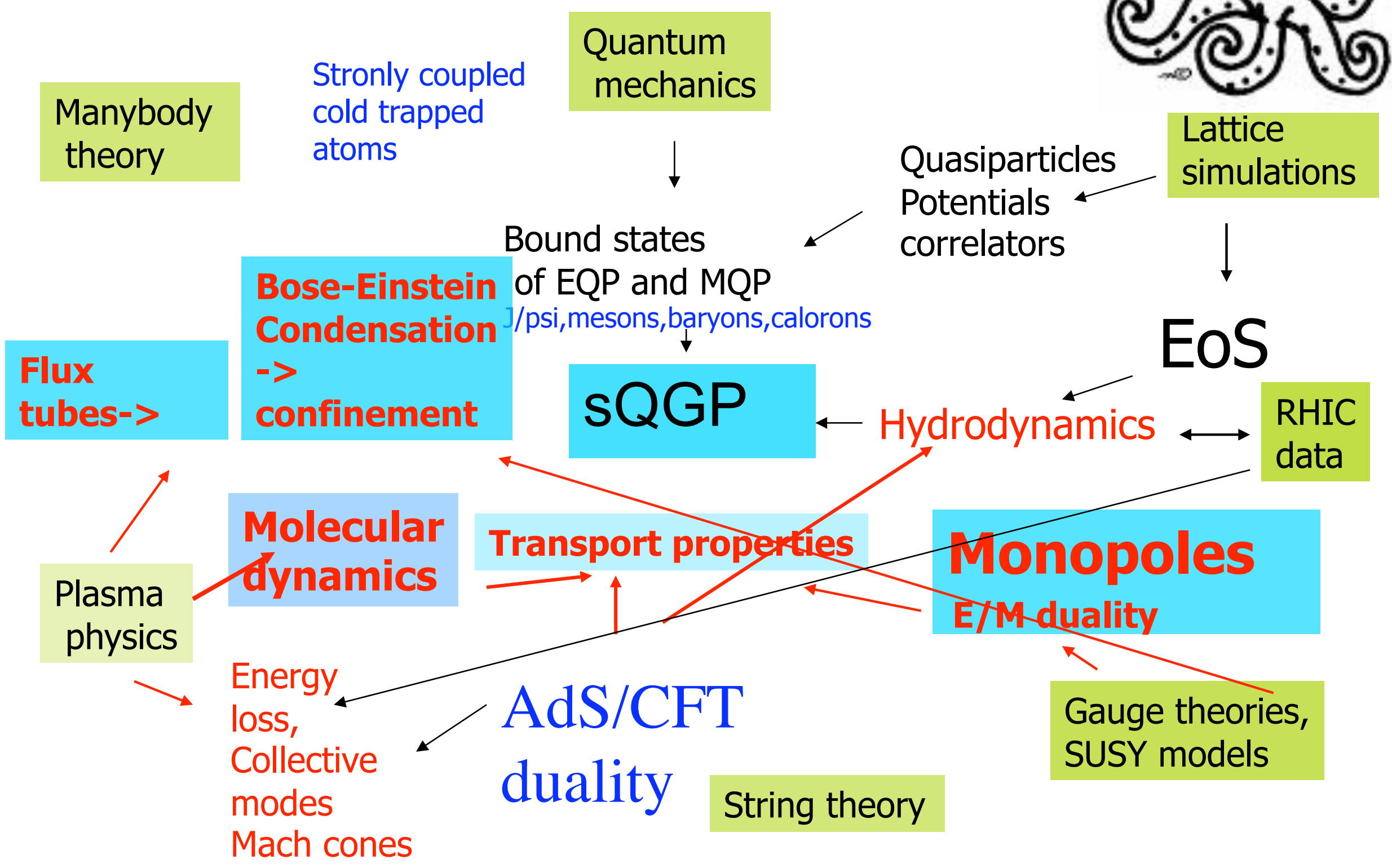




\section{The talk has many pictures but not so much text and equs: one may find some of them in archive 0807.3033}

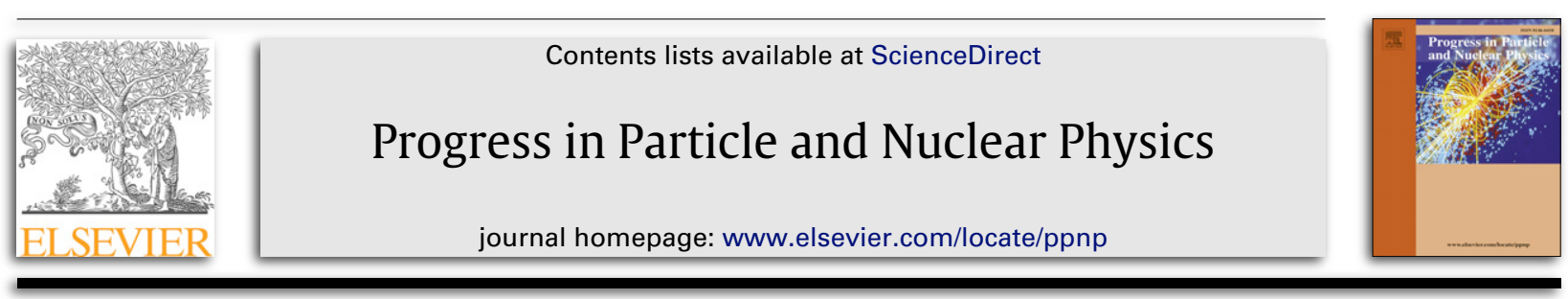

Review

Physics of strongly coupled quark-gluon plasma

Edward Shuryak

Department of Physics and Astronomy, University at Stony Brook, NY 11794, USA

\section{A R T I C L E I N F O}

Keywords:

Quark-gluon plasma

Finite temperature QCD

Heavy-ion collisions

AdS/CFT

\section{A B S T R A C T}

This review covers our current understanding of strongly coupled Quark-Gluon Plasma (sQGP), especially theoretical progress in: (i) explaining the RHIC data by hydrodynamics;

(ii) describing lattice data using electric-magnetic duality; (iii) understanding of gaugestring duality known as AdS/CFT and its application for "conformal" plasma. In view of the interdisciplinary nature of the subject, we include a brief introduction into several topics "for pedestrians". Some fundamental questions addressed are: Why is sQGP such a good liquid? What is the nature of (de)confinement and what do we know about "magnetic" objects creating it? Do they play any important role in sQGP physics? Can we understand the AdS/CFT predictions, from the gauge theory side? Can they be tested experimentally? Can AdS/CFT duality help us understand rapid equilibration/entropy production? Can we work out a complete dynamical "gravity dual" to heavy ion collisions?

(c) 2008 Elsevier B.V. All rights reserved. 


\section{Deconfinement/the energy density}

Is the transition 20 or $200 \mathrm{MeV}$ wide?

Bazavov et al, (HotQCD Coll.), arXiv:0903.4379
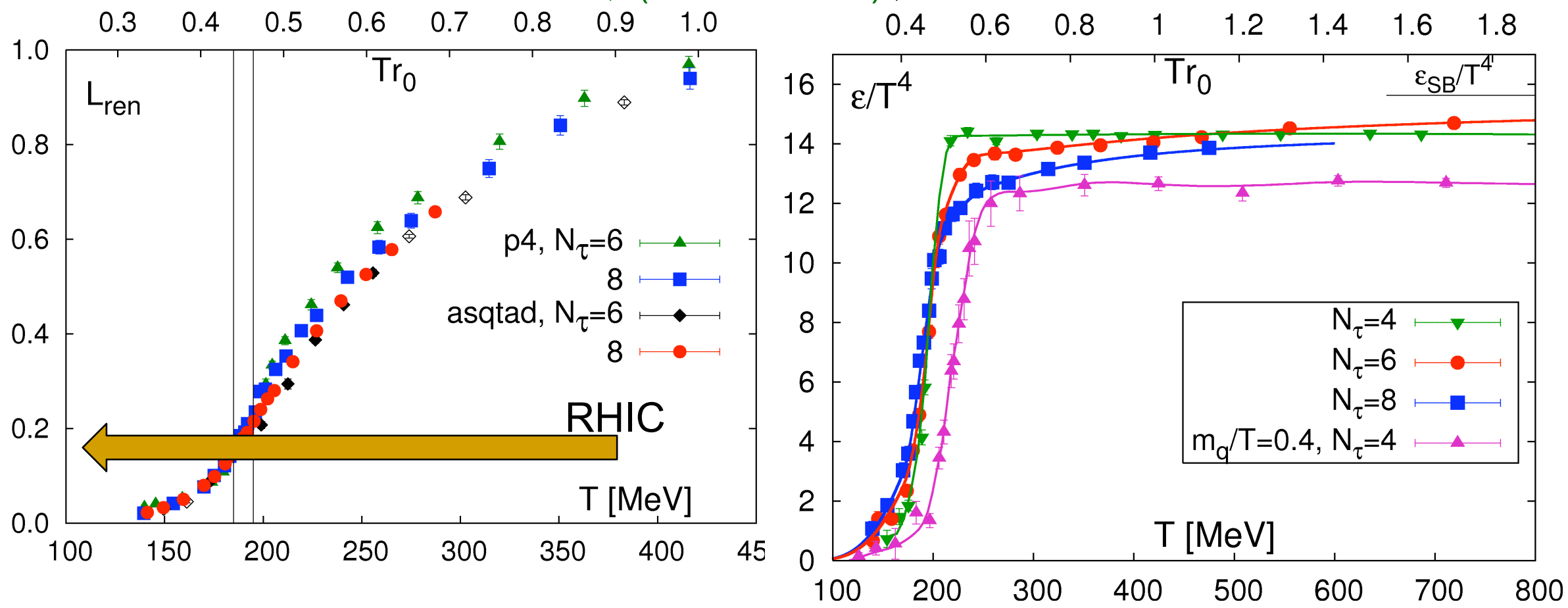

Renormalized Polyakov loop

QCD "Higgsing"=> nonzero $A_{0}=>$ imaginary chemical potential for electric charge -Quark density must be proportional to L (well checked using quark susceptibilities by Ratti,Weise, 06) : gluons to $L^{2}$ -Pisarski called the region $\mathrm{T}=(1-2) \mathrm{Tc}$ semi-QGP -And yet, no such suppression in entropy and energy density: what is missing there? <= Magnetic objects, unsuppressed by $L$ 
Magnetic objects and their dynamies: classies
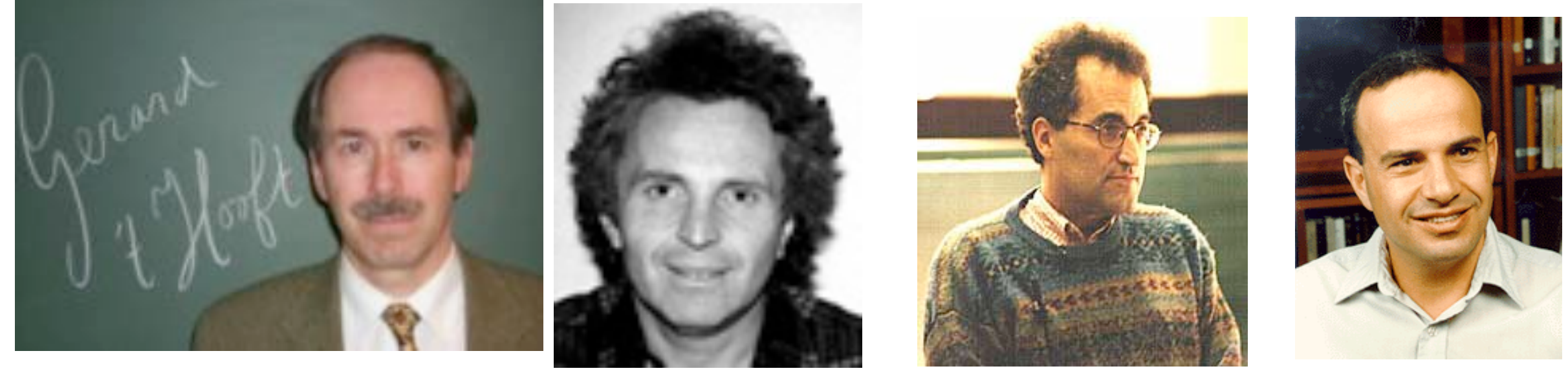

- Dirac explained how magnetic charges may coexists with quantum mechanics (1934)

- 't Hooft and Polyakov discovered monopoles in NonAbelian gauge theories (1974)

- "t Hooft and Mandelstamm suggested "dual superconductor" mechanism for confinement (1982)

- Seiberg and Witten shown how it works, in the $N=2$ Super - Yang-Mills theory (1994) 


\section{ㅂecectric and magnetic screening}

\section{masses (inverse screening lengths) from numerical simulation in lattice gauge theory Nakamura et al, 2004}

$$
\text { the "self-dual" E=M point }
$$

$\mathrm{Me}<\mathrm{Mm}$

Magnetic

Dominated

At $\mathrm{T}=0$ magnetic Screening mass Is about $2 \mathrm{GeV}$ (de Forcrand et al) (a glueball mass)

(Other lattice data -Karsch et alshow how Me Vanishes at Tc in more detail)

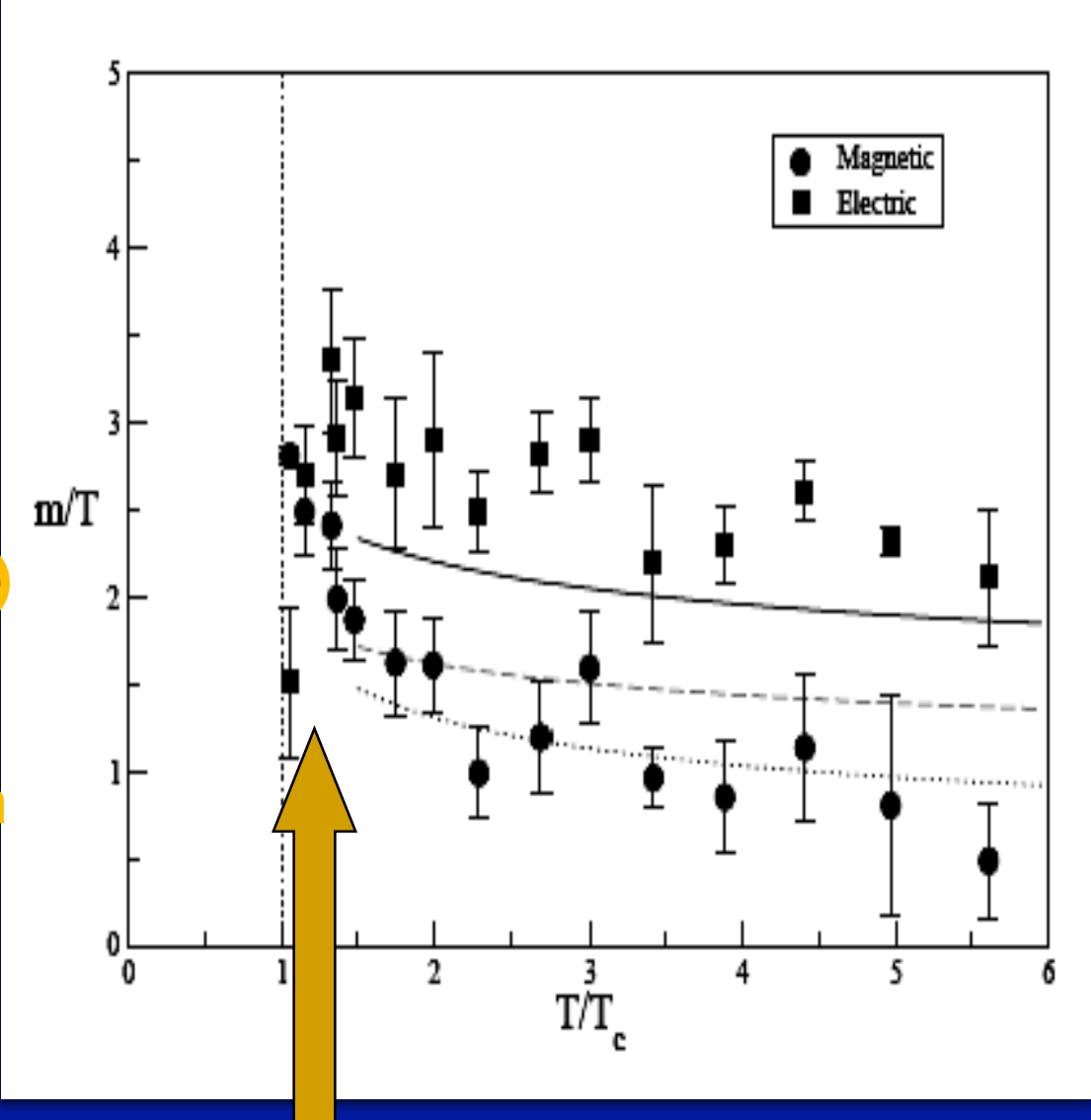

Me $>$ Mm

Electrric dominated

$\mathrm{M}_{\mathrm{E}} / \mathrm{T}=\mathrm{O}(\mathrm{g})$ ES 78 $\mathrm{M}_{\mathrm{M}} / \mathrm{T}=\mathrm{O}\left(\mathrm{g}^{\wedge} 2\right)$ Polyakov 79 Is QGP really getting magnetic as T<1.4Tc? 
"magnetic scenario": Liao,ES hep-ph/0611131,Chernodub+Zakharov Old good Dirac condition

\section{$\alpha_{\mathrm{s}}$ (electric) $\alpha_{\mathrm{s}}$ (magnetic) $=1$}

\section{$\Rightarrow$ electrie/magnetic couplings (e/g) must run in the opposite directions!}

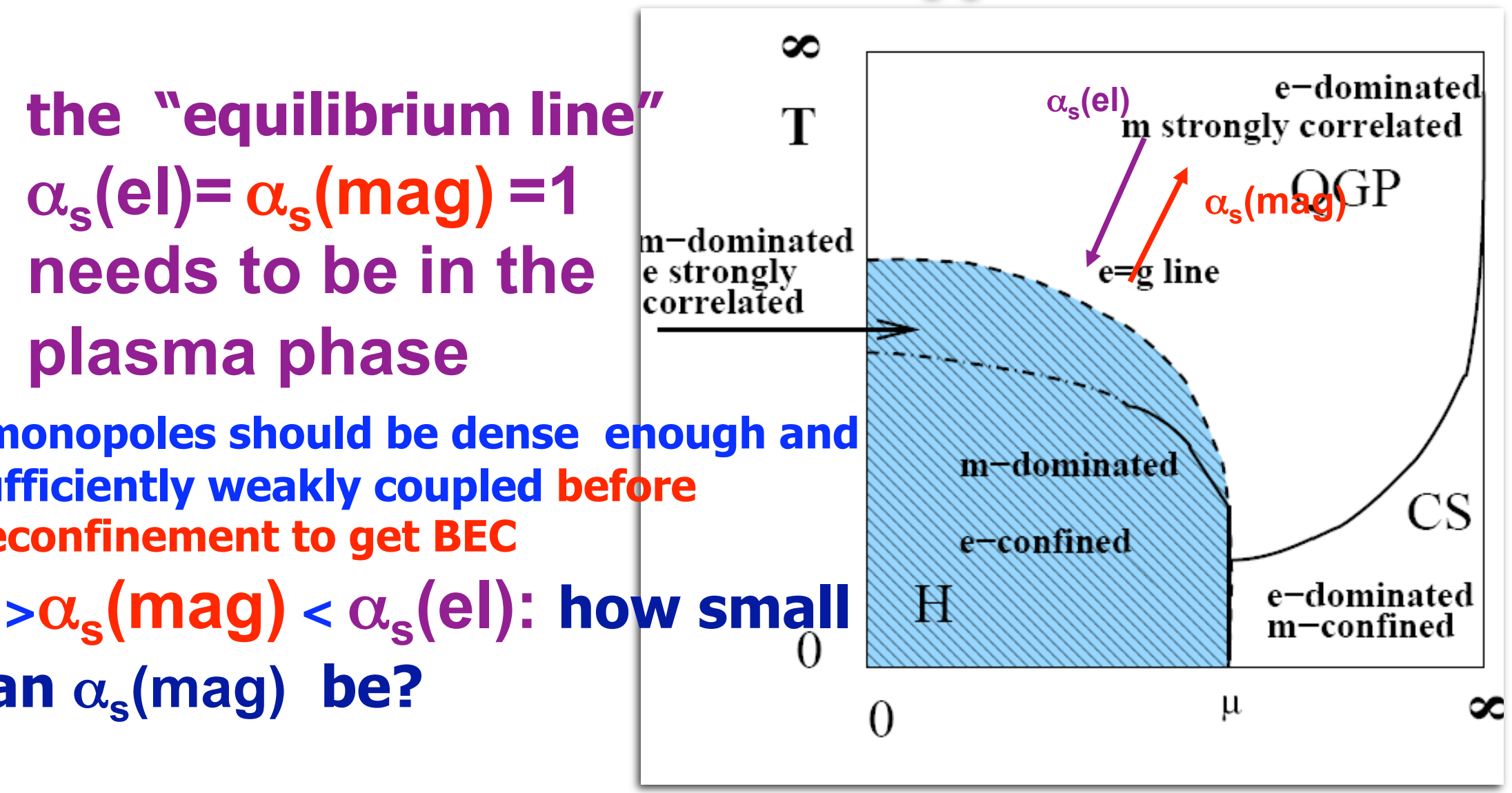




\section{Which magnetic objects are seen on the lattice?}

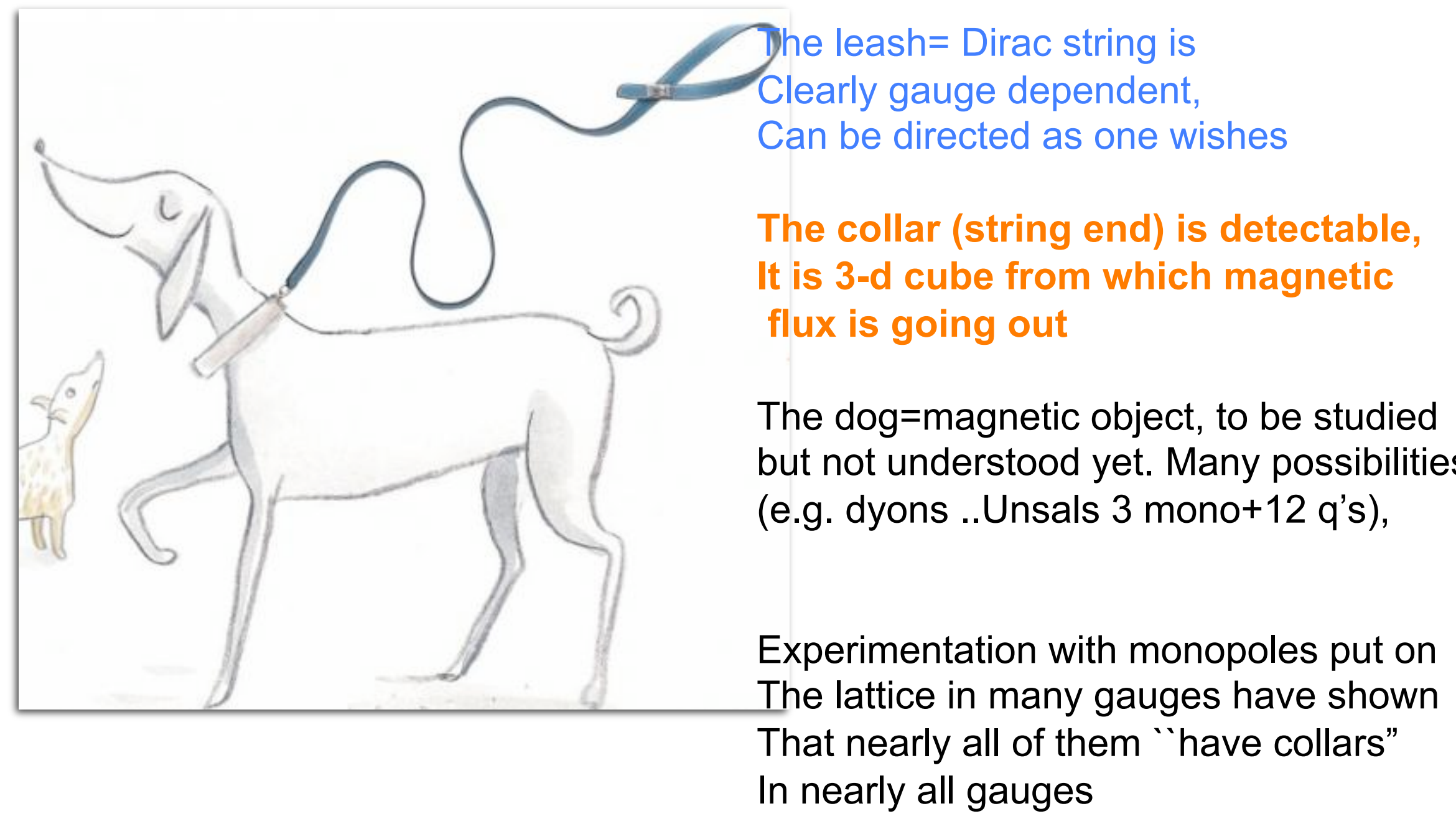


Magnetic monopoles in the high temperature phase of Yang-Mills theories

\section{Spring 2008 A. D'Alessandro and M. D'Elia'}

Dipartimento di Fisica, Universitd di Genova and INFN, Sexione di Genova,

Via Dodecaneso 39, I-16146 Genova, Italy

x-Correlations show it is a liquid $\Rightarrow$ We extract



FIG. 3. $\rho(T) / T^{3}$ as a function of $T / T_{c}$. Data have been obtained on a $48^{3} \times L_{t}$ lattice, with variable $L_{t}$ and at $\beta=2.75$ (first 9 points), and variable $\beta$ at $L_{l}=4$ (last 10 points). magnetic

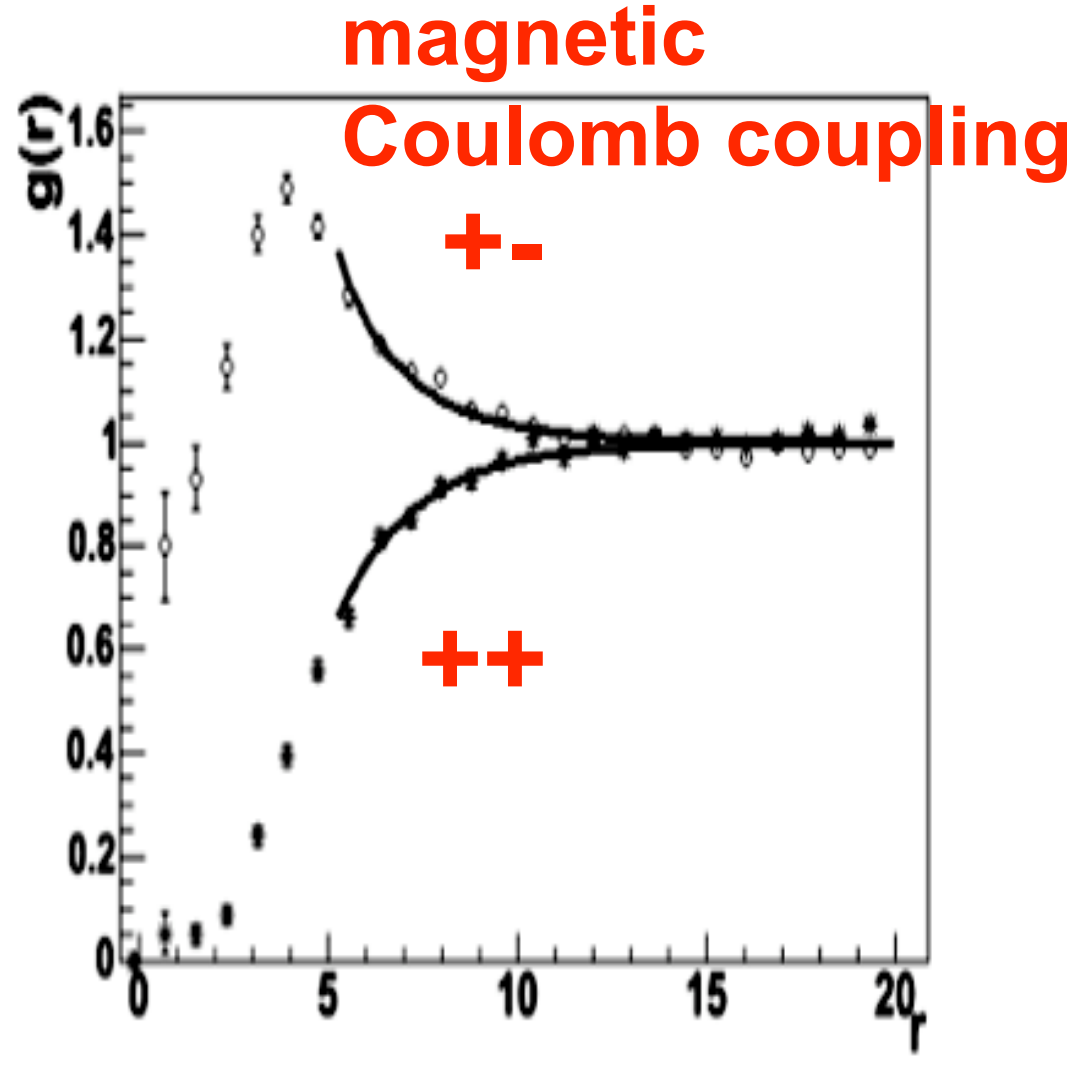

FIG. 5. $g(r)$ for the monopole-monopole (stars) and monopole-antimonopole (circles) case on $0^{3} \times 5$ lattice at $\beta=2.7\left(T \simeq 2.85 T_{c}\right)$. The reported curves correspond to fits according to )$=\exp (-V(r) / T)$ with $V(r)$ a Yukawa potential (see Eqs. (2.9) and $(2.10))$.

Lattice SU(2) gauge theory, monopoles found and followed by Min.Ab.gauge 


\section{Magnetic Component of Quark-Gluon Plasma is also a Liquid!}

Jinfeng Liao and Edward Shuryak

Department of Physics and Astronomy, State University of New York, Stony Brook, NY 11794

(April 1, 2008)

The so called magnetic scenario recently suggested in [1] emphasizes the role of monopoles in strongly coupled quark-gluon plasma (sQGP) near/above the deconfinement temperature, and specifically predicts that they help reduce its viscosity by the so called "magnetic bottle" effect. Here we present results for monopole-(anti)monopole correlation functions from the same classical molecular dynamics simulations, which are found to be in very good agreement with recent lattice results [2]. We show that the magnetic Coulomb coupling does run in the direction opposite to the electric one, as expected, and it is roughly inverse of the asymptotic freedom formula for the electric one. However, as $T$ decreases to $T_{c}$, the magnetic coupling never gets weak, with the plasma parameter always large enough $(\Gamma>1)$. This nicely agrees with empirical evidences from RHIC experiments, implying that magnetic objects cannot have large mean free path and should also form a good liquid

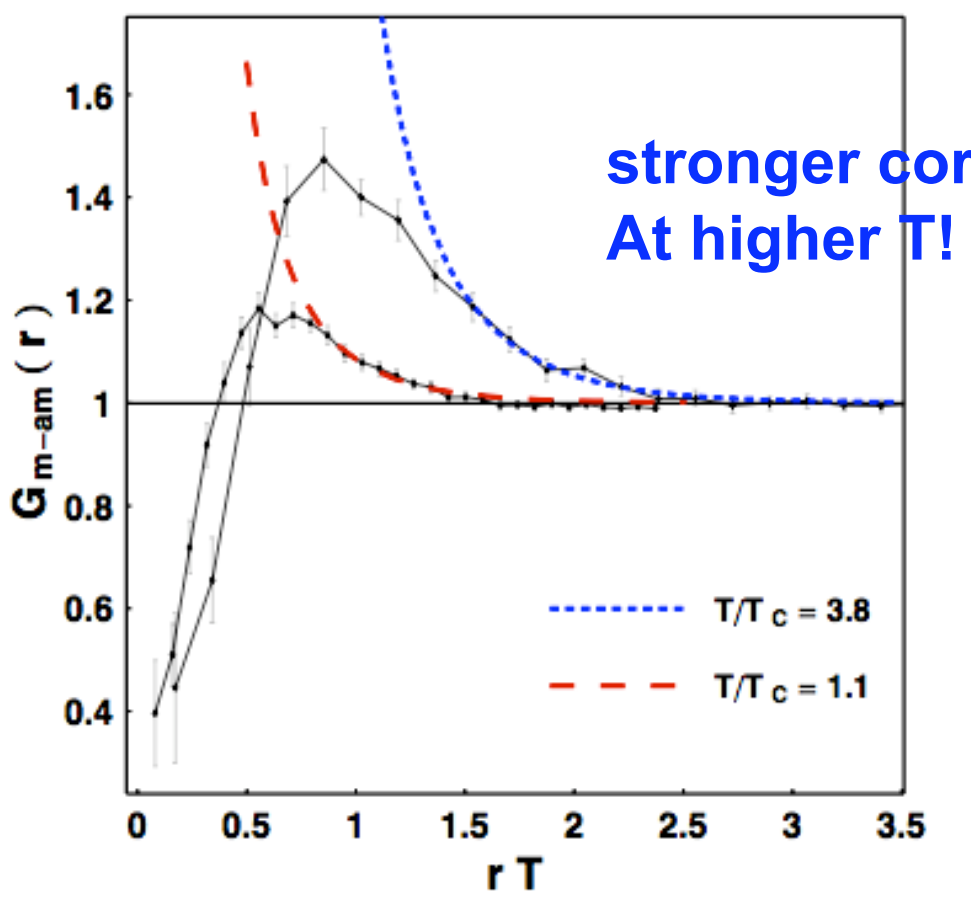

FIG. 2. (color online) Monopole-antimonopole correlators versus distance: points are lattice data [2], the dashed lines are our fits.

Our MD for 50-50 MQP/EQP

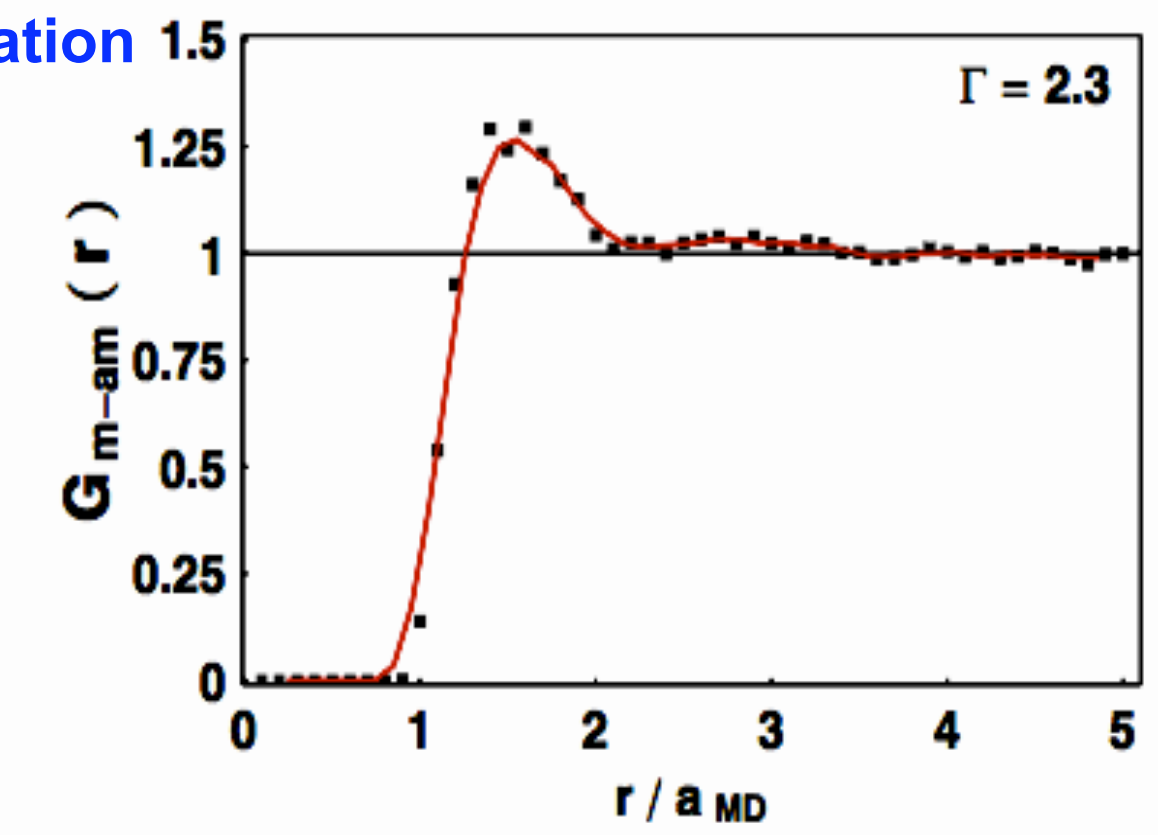




\section{$\alpha_{s}$ (electrie) and $\alpha_{s}$ (magnetic) \\ do run in opposite directions!}

- Squares: fitted magnetic coupling, circles: its inverse compared to asymptotic freedom (deasifinedl)

- Effective magnetic plasma parameter $\Gamma \equiv \frac{\alpha_{C} /\left(\frac{3}{4 \pi n}\right)^{1 / 3}}{T}$

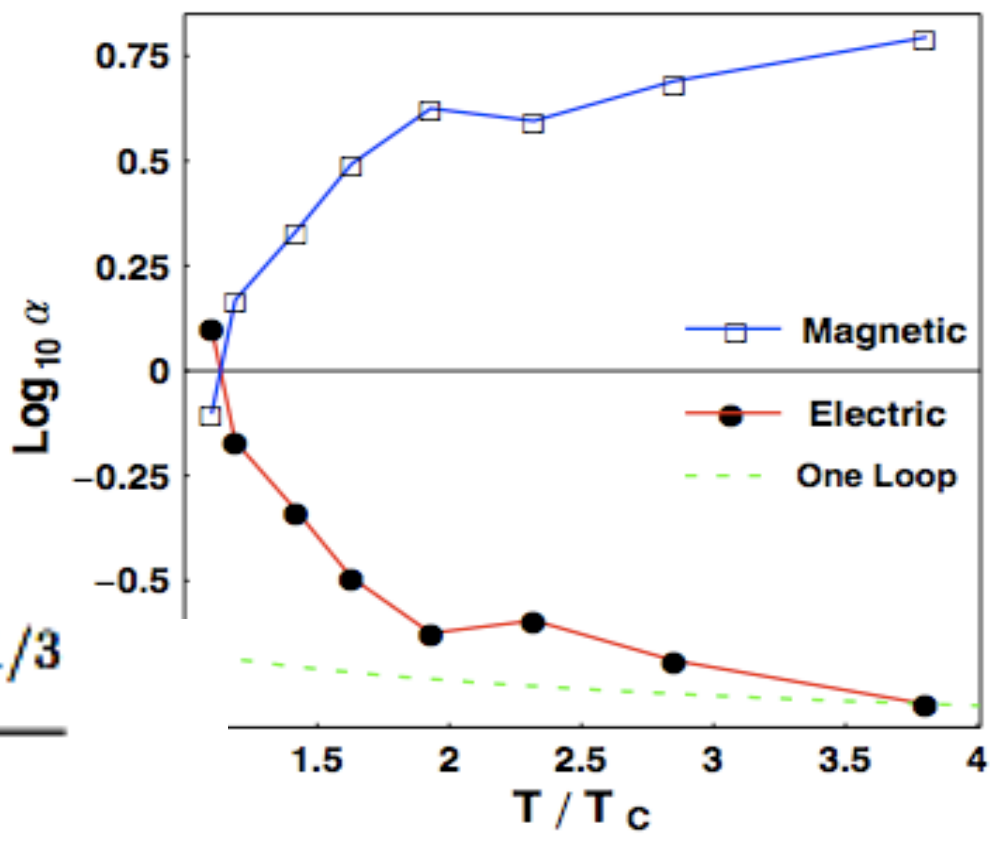

- So, the monopoles are never weakly coupledl!

- (just enough to get Bose-condenced, see later)

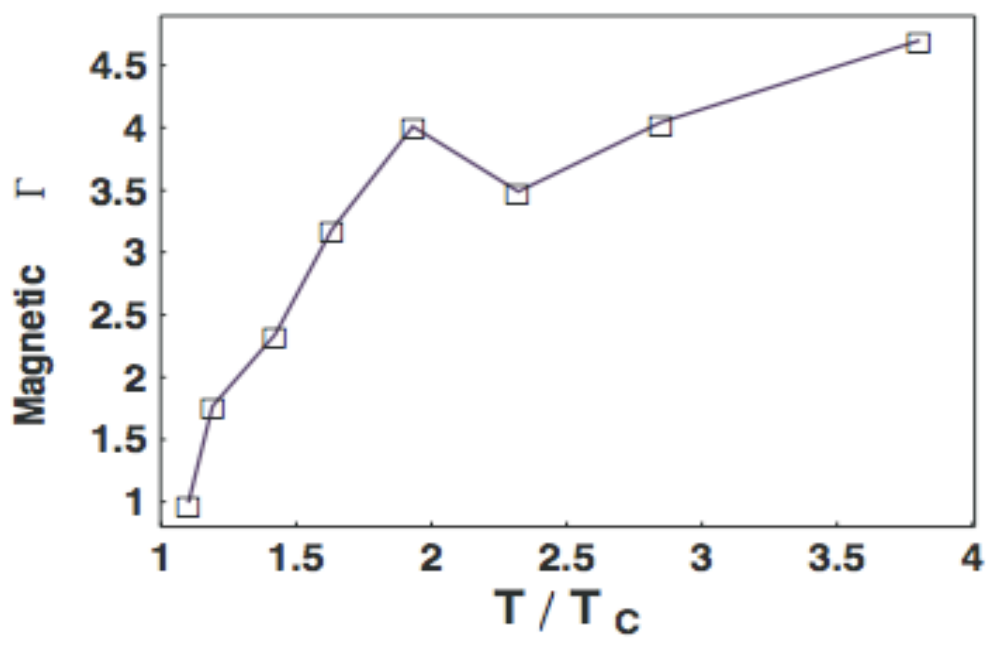




\section{" caging" and (classical MD) transport}
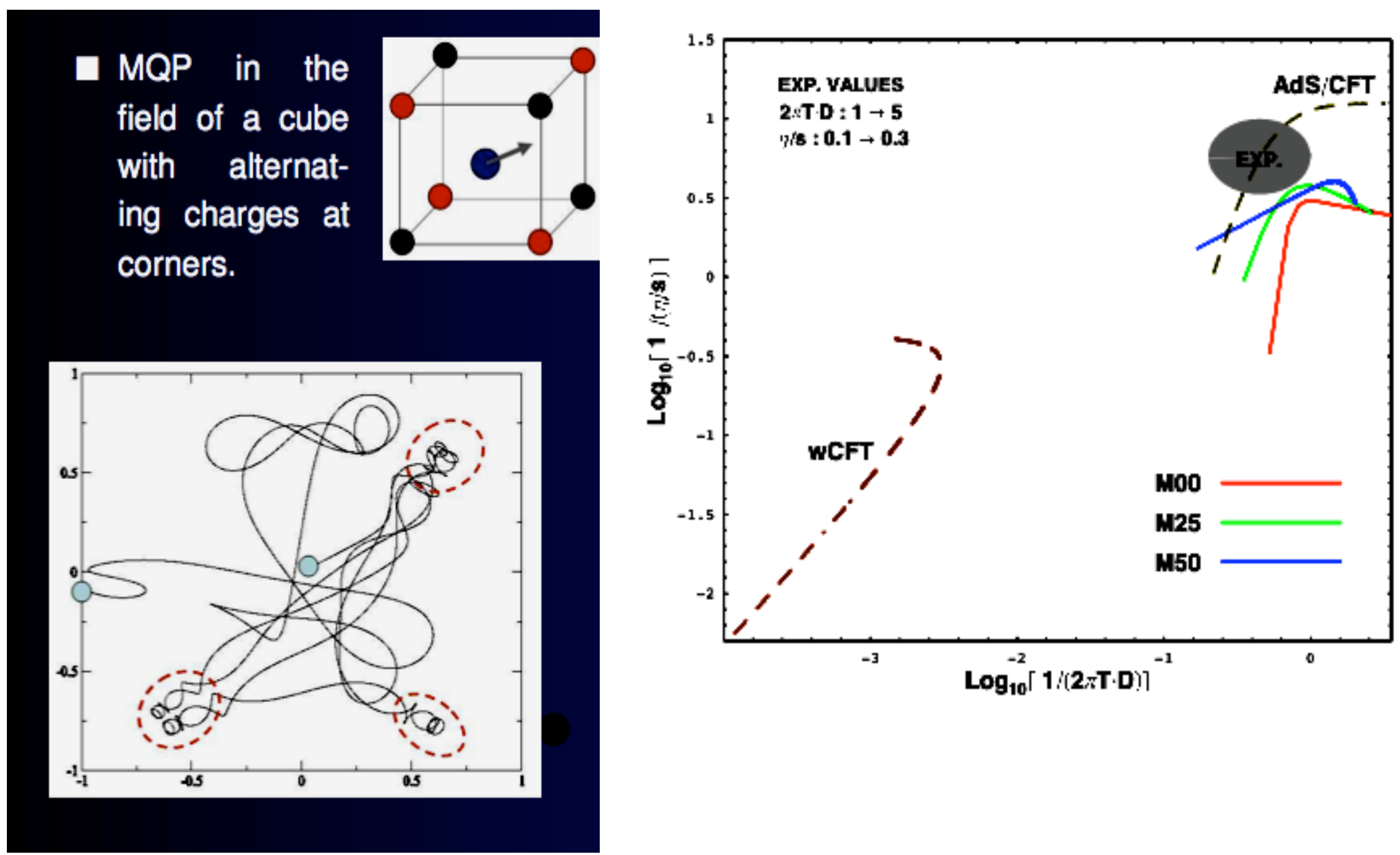
Monopoles in electric Quark-Gluon Plasma

$$
\begin{gathered}
\text { Claudia Ratti and Edward Shuryak } \\
\text { Department of Physics and Astronomy } \\
\text { University at Stony Brook,NY } 11794 \text { USA } \\
\text { shuryak@tonic.physics.sunysb.edu }
\end{gathered}
$$

November 24, 2008

- Quantum problem of gluon-monopole scattering

- $n=e g(=1)$ is the only parameter, if we ignore the monopole core and keep only Coulomb B field

We denote the vector harmonics by $\Phi_{j, n}^{m, \sigma}(\theta, \varphi)_{a i}$. They obey the following eigenvalue equations

$$
\begin{aligned}
& \left\{\begin{array}{l}
\vec{J}^{2} \\
J_{3} \\
(\hat{r} \cdot \vec{I}) \\
(\hat{r} \cdot \vec{S})
\end{array}\right\} \Phi_{j, n}^{m, \sigma}(\theta, \varphi)_{a i}=\left\{\begin{array}{l}
j(j+1) \\
m \\
n \\
\sigma
\end{array}\right\} \Phi_{j, n}^{m, \sigma}(\theta, \varphi)_{a i} . \\
\phi^{(+)}(\vec{r})= & e^{-i \pi n} \sum_{j=|n|}^{j_{\max }}(2 j+1) e^{i \pi j} e^{-i \pi j^{\prime} / 2} j_{j^{\prime}}(k r)\left[U(-\varphi, \theta, \varphi) \chi_{i}^{n}\right] \mathcal{D}_{n,-n}^{(j)}(-\varphi, \theta, \varphi) \\
= & {\left[U(-\varphi, \theta, \varphi) \chi_{i}^{n}\right] \psi^{(+)}(\vec{r}) . }
\end{aligned}
$$

We recall that the index $j^{\prime}$ in the above formula is the positive root of

$$
j^{\prime}\left(j^{\prime}+1\right)=j(j+1)-n^{2} .
$$




\section{A surprize: no corrections to thermodynamies}

- Beth-Uhlenbeck

$\delta M_{m}=\frac{T}{\pi} \sum_{j}(2 j+1) \int d k \frac{d \delta_{j}}{d k} f(k, T)$ correction (extra states in a box) is zero because there is no dependence on $k$

The origin of this somewhat unexpected result can be traced to the fact that the Beth-Uhlenbeck expression was derived from a semiclassical counting of the density of states in a large (spherical) box containing the monopole. However the semiclassical density of states, related to classical phase space, is insensitive to magnetic fields because the corresponding integral

$$
\Omega_{c l}(E)=\int \frac{d^{3} p d^{3} x}{(2 \pi)^{3}} \delta(E-H(p, x))
$$

for an electric particle in any magnetic field $H=(\vec{p}-e \vec{A}(x))^{2} / 2 m$ does not depend on the field at all (in order to see that this explanation is correct, consider for 


\section{Seattering amplitude}

vs maximal impact parameter or $\mathbf{j}_{\max }$

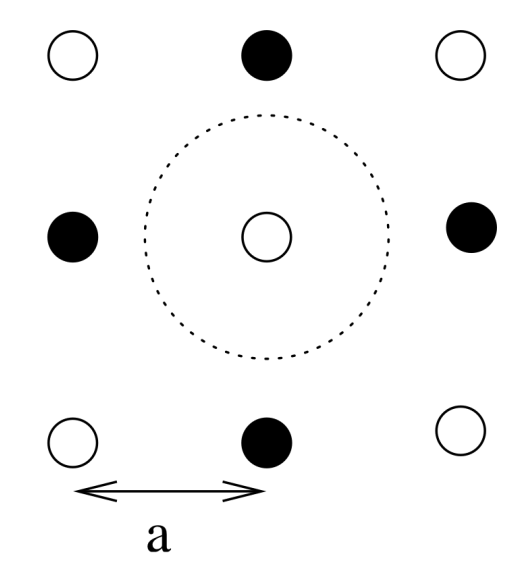

Transport cross section: results
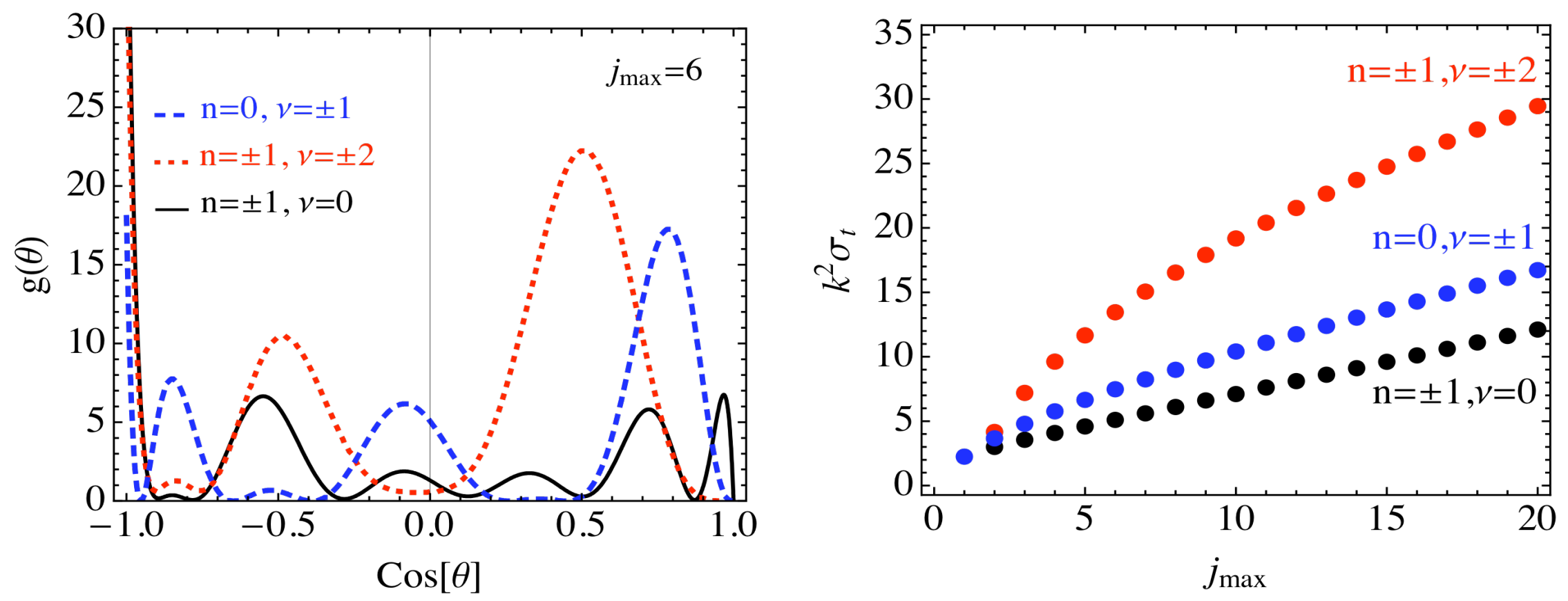

$$
g(\theta)=(1-\cos \theta)|f(\theta)|^{2}
$$




\section{Not surprising, large correction to transport}
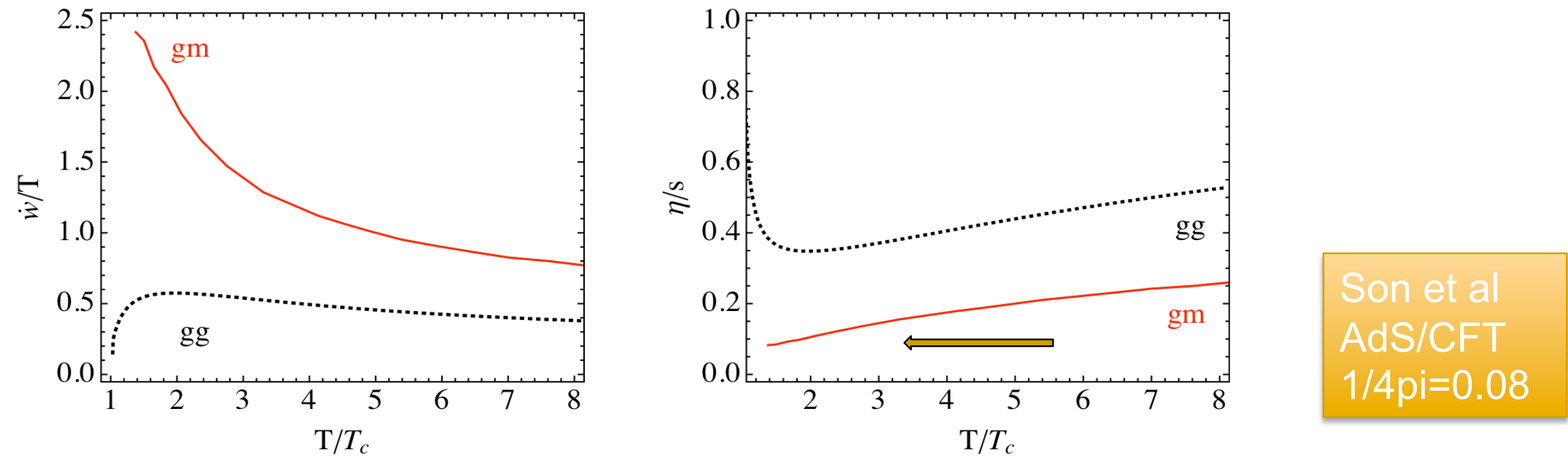

Figure 14: Left panel: gluon-monopole and gluon-gluon scattering rate. Right panel: gluon-monopole and gluon-gluon viscosity over entropy ratio, $\eta / s$.

\section{- RHIC: T/Tce2, LHC T/TGeS; we predict hydro will still be there, with $\eta / s$ about .2}




\section{Reviving Feynman theory of BEC for He4}

- Feynman (1950's): sum over the polygon jumps diverges when $\Delta \mathbf{S}$ (jump)<Sc universally!

$$
Z=\sum_{k} C_{k}(\text { geometr }) \exp (-k \Delta S)=\sum_{k}\left(c e^{-\Delta S}\right)^{k} \underbrace{}_{-\ldots}
$$

PIMC done in 1980-1990 and is still going (Ceperley, RMP) explained many things accurately, still not elucidated if Feynman was completely right 


\title{
Bose-Einstein Condensation of strongly interacting bosons: from liquid He4 to QCD monopoles
}

\author{
Marco Cristoforetti ${ }^{1}$ and Edward V. Shuryak ${ }^{2}$
}

${ }^{1}$ Physik Department, Technische Universität München, D-85747 Garching, Germany

${ }^{2}$ Department of Physics and Astronomy, State University of New York, Stony Brook NY 11794-3800, USA

(Dated: April 8, 2009)

Starting from classic work of Feynman on the $\lambda$-point of liquid Helium, we have developed a simple "moving string model" which can rather accurately predict the critical temperature for liquid He4. It approximates Feynman's "polygons" by an infinite line of particles, simultaneously jumping to its neighbor's place. The action needed for that per atom is calculated both semiclassically and numerically: we then follow Feynman's idea that this action should be some universal constant. This treatment also explains why high density (solid) He is not a BEC state. We then use this model for deconfinement phase transition of QCD-like gauge theoris, relating it to BEC of (color)magnetic monopoles. We start with Feynman-like approach without interaction, estimating the monopole mass at $T_{c}$. Then we discuss the role of monopole's Coulomb repulsion, and end up proposing certain lattice studies of monopole "polygons" (or clusters).
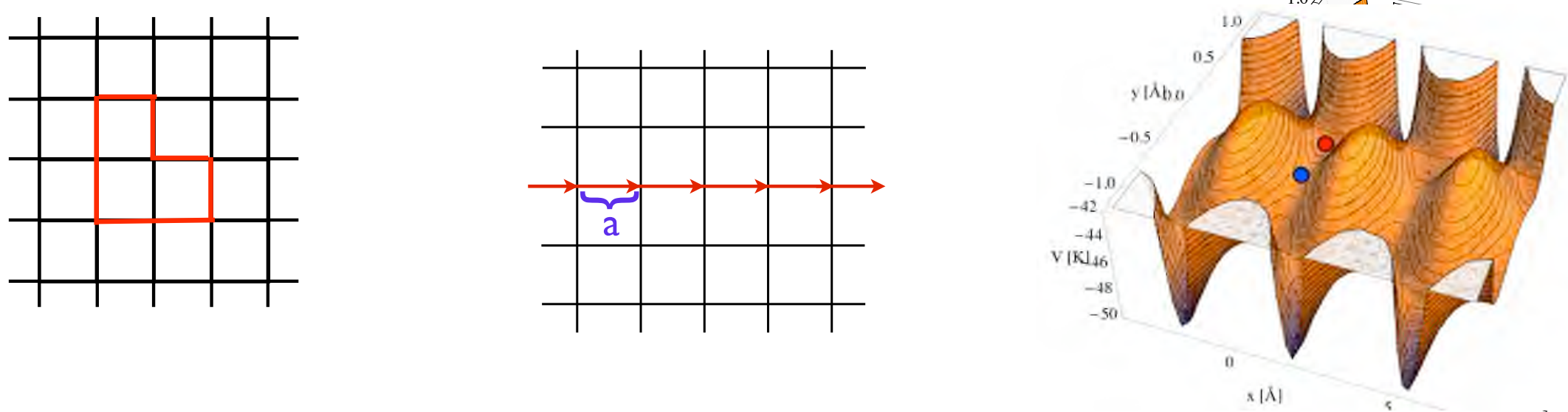

\section{Large polygons $\Rightarrow$ moving line model studied semiclassically and by the $1 d$ path integral numerically}




\section{Making instantons practical,}

\section{Once again}

$$
\begin{aligned}
& T_{c}\left[d, V_{0}, E_{E}\right]=\left(\sqrt{\frac{m}{2 E_{E}}} \frac{d}{\pi} \mathrm{F}\left[\pi,-V_{0} / E_{E}\right]\right)^{-1} \\
& \left.=\sqrt{\frac{m}{2 E_{E}}} \frac{d}{\pi} 2 \mathrm{~K}\left[-V_{0} / E_{E}\right]\right)^{-1} .
\end{aligned}
$$

provided the energy is substituted from the Feynman condition, which say that the Euclidean action

$$
\begin{aligned}
& S_{E}\left[d, V_{0}, E_{E}\right]=\int_{0}^{\beta} \mathrm{d} \tau\left(\frac{m}{2} \dot{x}_{c l}^{2}+V(x)\right) \\
& =\frac{d}{\pi} \sqrt{\frac{m E_{E}}{2}} 2\left(2 \mathrm{E}\left[-\frac{V_{0}}{E_{E}}\right]-\mathrm{K}\left[-\frac{V_{0}}{E_{E}}\right]\right),
\end{aligned}
$$
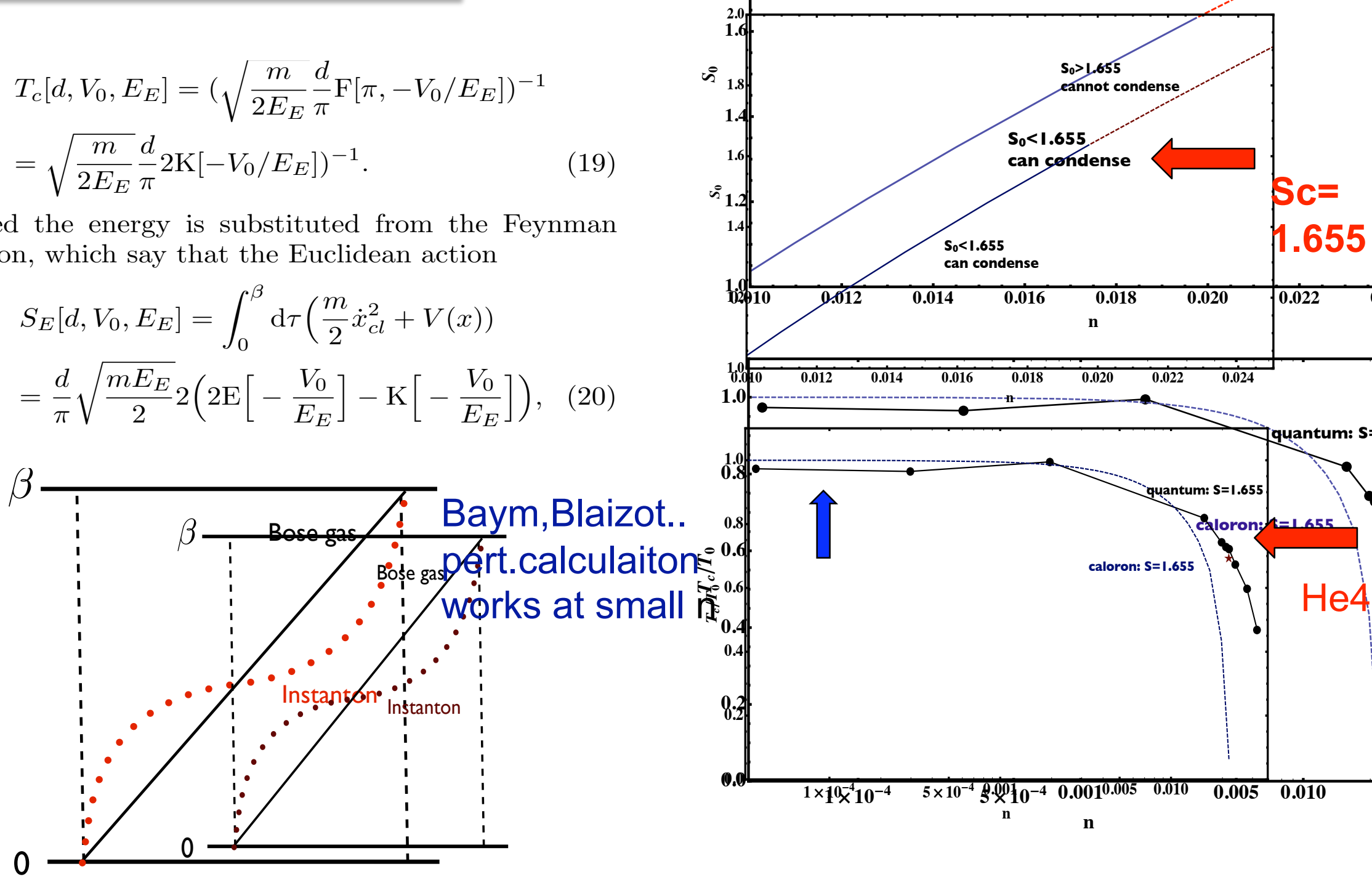

FIG. 10: (a) The action for the instanton solution as a function of the density. When the action is larger than the critical value $S=1.655$ (dashed line) the system cannot be Bose-Einstein condensed phase.

(b) The critical temperature obtain from the caloron/instanton solution (dashed line) and from the 1-d PIMC simulation (points). The red star shows the 


\section{As density grows, Feynman condition cannot be satisfied: $=>$ solid $\mathrm{He} 4$ is not supersolid}
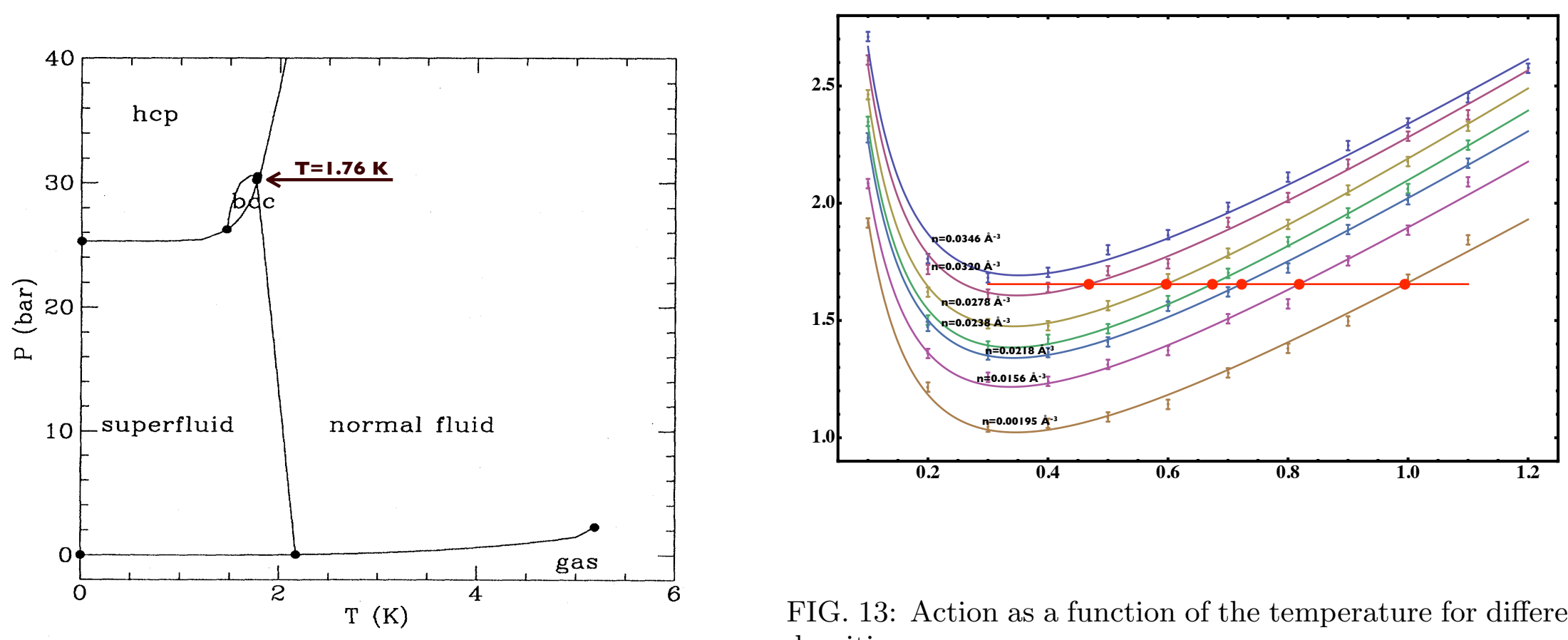

FIG. 13: Action as a function of the temperature for different densities. 


\section{BEC (confinement) condition for monopoles}

For charged Bose gas (monopoles) the action for the jump can be calculated similarly, but relativistically; jumps in space $d$ and in time Comparable)

$\Delta S=M$ sqrt $\left(\mathrm{d}^{2}+(1 / \mathrm{Tc})^{2}\right)+\Delta \mathrm{S}($ interaction $)=\mathrm{Sc}$ $=1.655$

$=>$ Upper bound on the monopole mass $\mathrm{M}$ at Tc $M(T C)<300 \mathrm{MeV}$ 


\section{Lattice studies of monopole clusters near Te (M.D'Ellia,A.D'Alessandro)}

$$
\mu(T)=\log \left[\left(\frac{1}{2}\right)^{5 / 2} \frac{n_{1}(T)}{n_{2}(T)}\right]
$$

or equivalently from the same (5) applied to trajectories wrapped twice $n_{2}$ and three times $n_{3}$

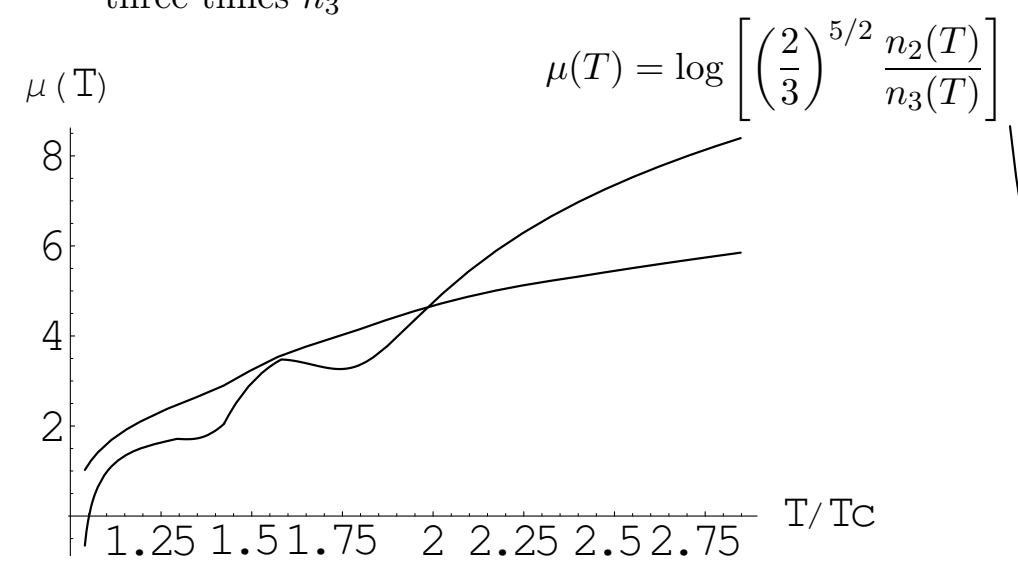

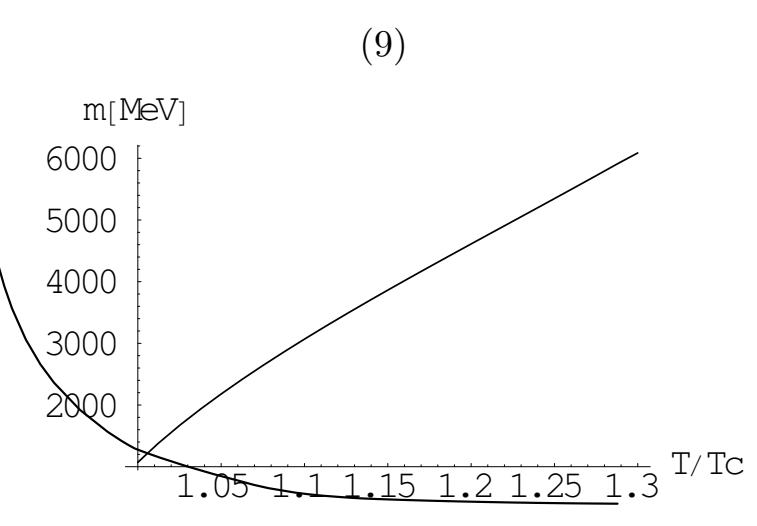

$\mathrm{M}(\mathrm{T}=\mathrm{Tc})=>.3 \mathrm{GeV}$. As predicted

$\begin{array}{cc}\mathrm{T} / \mathrm{Tc} & \mathrm{m}[\mathrm{GeV}]=1 /\left(2<x^{2}>T\right) \\ 1.095 & 0.617(16) \\ 1.187 & 0.907(16) \\ 1.295 & 1.229(29) \\ 1.424 & 1.671(12) \\ 1.582 & 2.163(19) \\ 1.780 & 2.736(15) \\ 2.035 & 3.360(14) \\ 2.374 & 4.047(13) \\ 2.848 & 4.856(11) \\ 7.000 & 15.59(2)\end{array}$

- Clusters with $\mathrm{k}<4$ behave like in $\mathrm{He}$

- mu and monopole masses consistently determined, they are lighter than dyons $(\mathrm{M}($ dyon $) / \mathrm{T}=\mathrm{S}$ (inst)/Nc=4) 
New phenomena in heavy ion collisions:

the "cone" and "ridges" are seen

they are "too bright" $\Rightarrow>$ flux tubes seem to survive

for long time 


\section{Three main jet correlations :}

- "Shoulder" on the away side => "conical flow"

(H.Stoecker,J.Casalderrey+ES, 2005)

- "Hard ridge" => forwardbackward bremmstrahlung cones kicked out by hydro radial flow

(ES 0706.3531, PRC76)

- "Soft ridge" => initial stage fluctuation of the color changes

(Dumitru,Gelis,McLerran,Venugopalan, 0804.3858, Gavin et al 0806.4718) 


\section{The 3-particle correlations confirmed conical shape. The cone angle seems to be universal,}

\section{but large $\Rightarrow e_{s}=.2$, why?}

Why come is so bright? and why there is mo wake?

Why does it exists at SPS? (CERES)
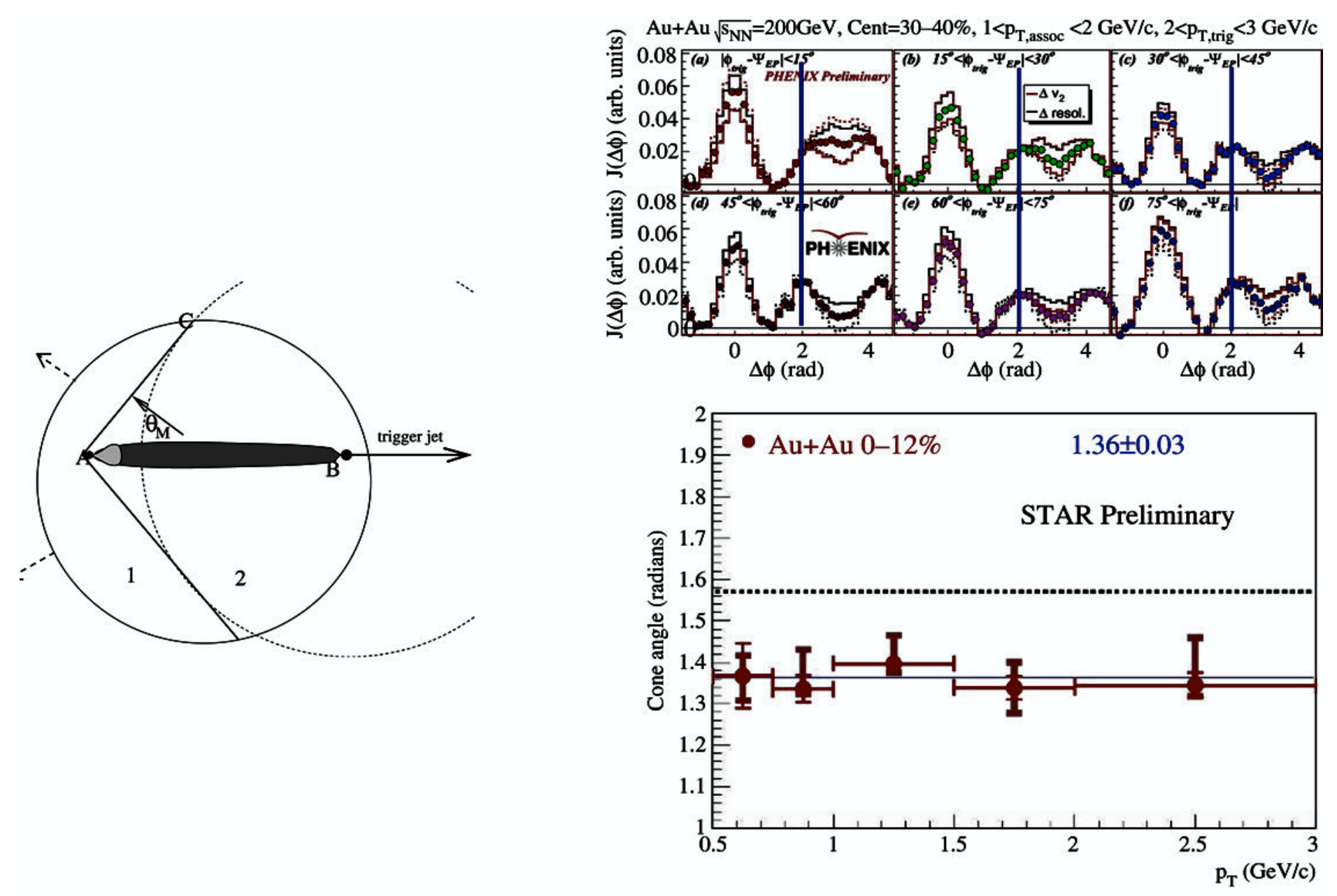


\section{STAR found that the "ridge" exists even without any hard trigger (=>good statitics)}

(Phobos further observed

that ridge extends at least till $|y|$ $=4$ )
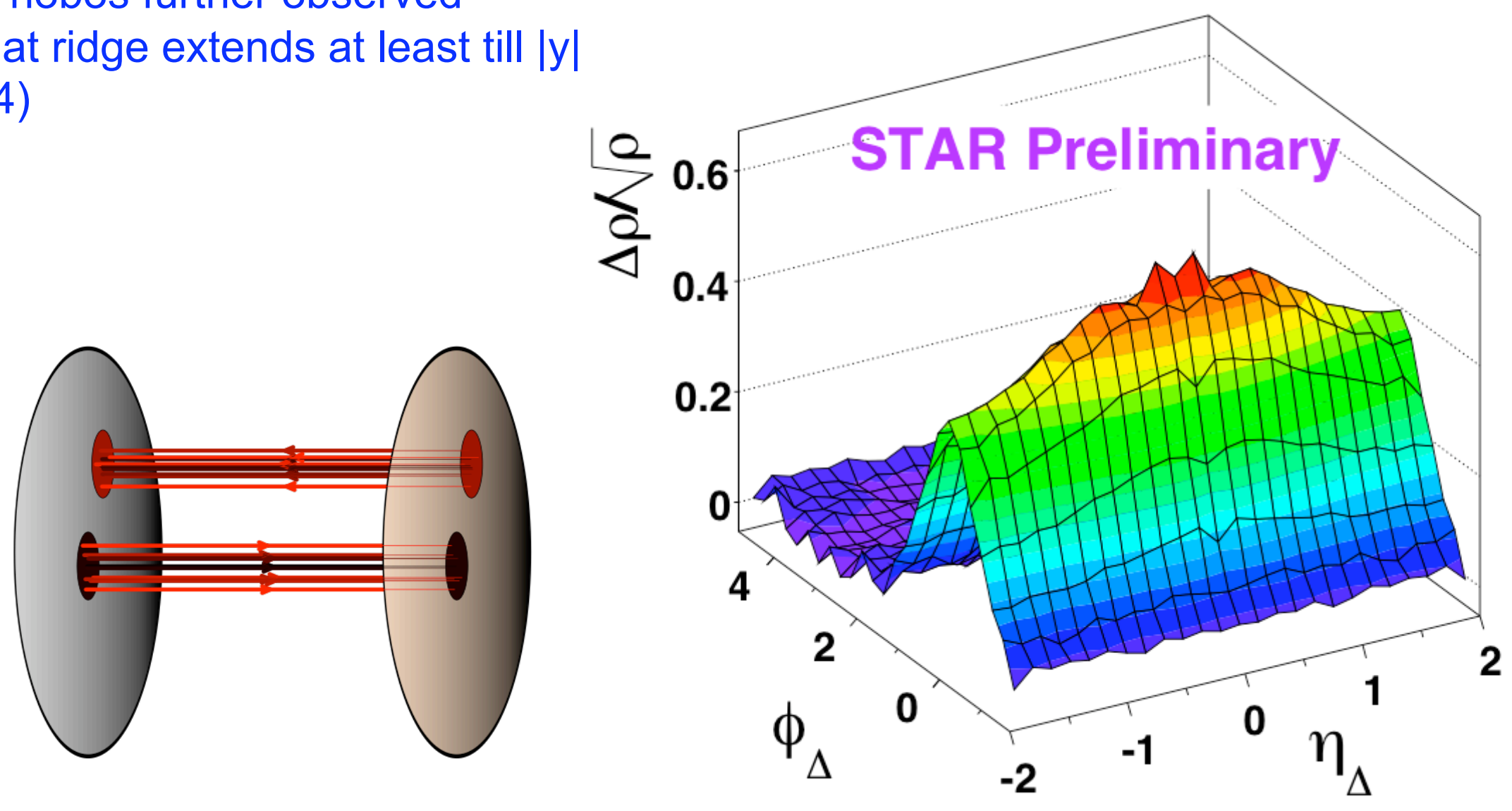
The Fate of the Initial State Fluctuations in Heavy Ion Collisions

We study the propagation of local density fluctuations created in heavy ion collisions (either due to hard collisions or color charge separation) through the hydrodynamical evolution, which is modeled by overall Hubble flow. While naively they should expand to spherical waves of sound with the radius given by sound horizon, making them delocalized and hardly observable, the real solution is much more interesting. The interplay of time-dependent speed of sound and Hubble expansion leads to recreation of a fluctuations at original location and even their amplification.

Naively, "spots" should excite a wave and get expanded to a spherical (or conical, or cylindrical) wave

Like in the case of stone thrown into the pond, nothing is left at the original position: so how can they be observed?

Its size $=>$ the sound horizon $=>$ is comparable to fireball size 6-8 fm/c

And thus large angular size $R_{h}=\int_{0}^{\tau_{f}} d \tau c_{s}(\tau)$

But the actual solution to the problem reveals creation of smaller-brighter second waves which we may see
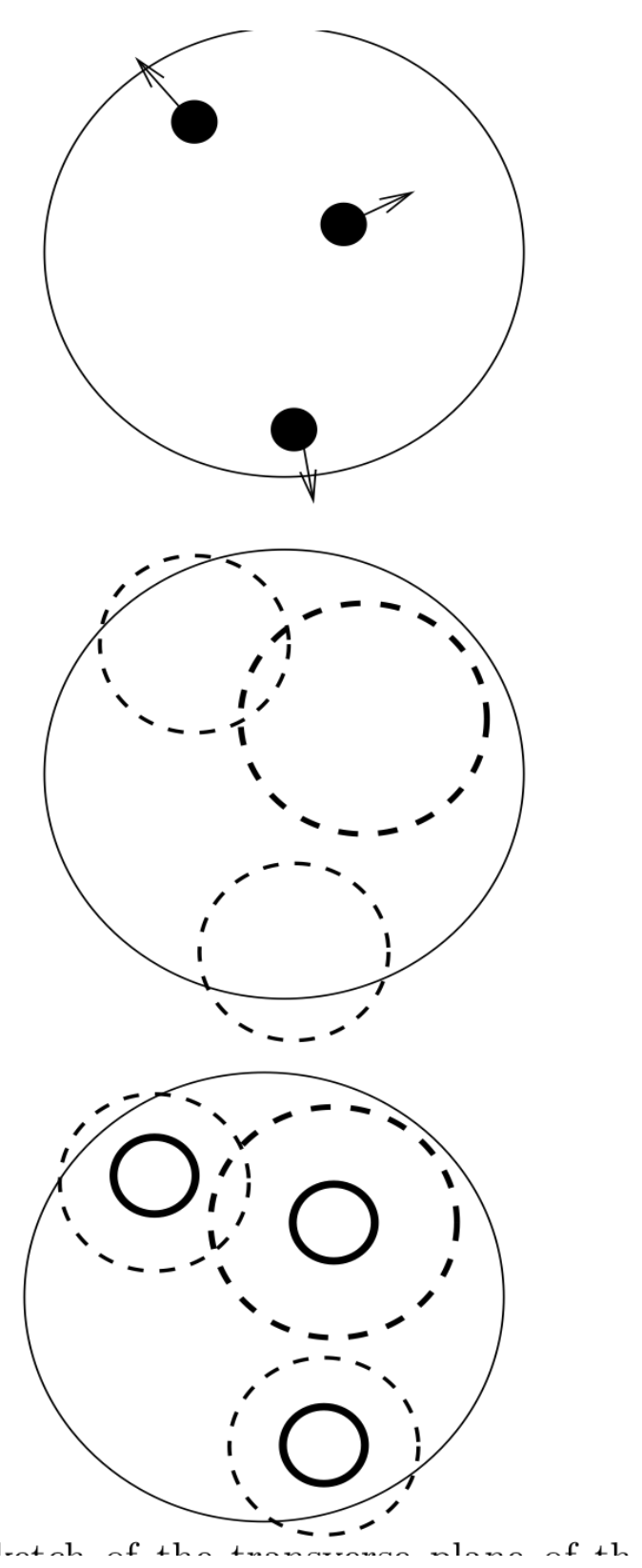
- Either "acoustic solution" : the second wave of sound due to rapid $\mathrm{c}_{\mathrm{s}}(\mathrm{t})$ dependence

- Or the flux tube solution : a "coil" preventing expansion of the (electric) flux tube

(possible if matter is basically magnetic plasma, with very few electric charges) 


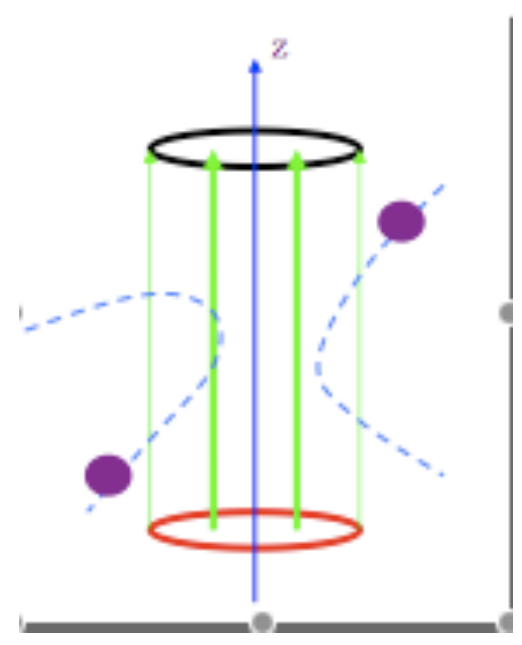

\section{e-flux tubes \\ above Tc?}

(with J.F.Liao, archive 0706.4465)

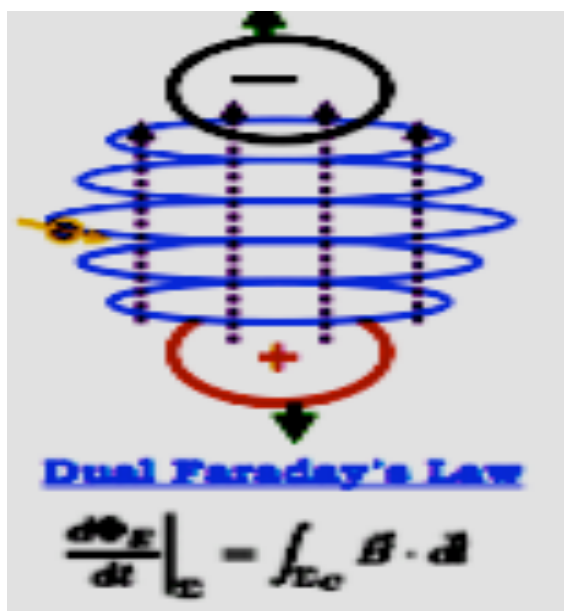

- Dual superconductivity at $T<T c$ as a confinement mechanism ('tHooft, Mandelstam 1980's) => monopole Bose condensation $=>$

electric flux tubes (dual to Abrikosov-Nielsson-Olesen vortices)

- Dual magnetohydrodynamics at $T>T C$ ? Electric flux tubes in magnetic plasma

- monopoles are reflected from $E$ field $=>$ pressure $\Rightarrow$ metastable flux tubes up to $1.4 \mathrm{Tc}$ 


\section{My favorite example of flux tubes without super/quantum}

- metastable with normal current
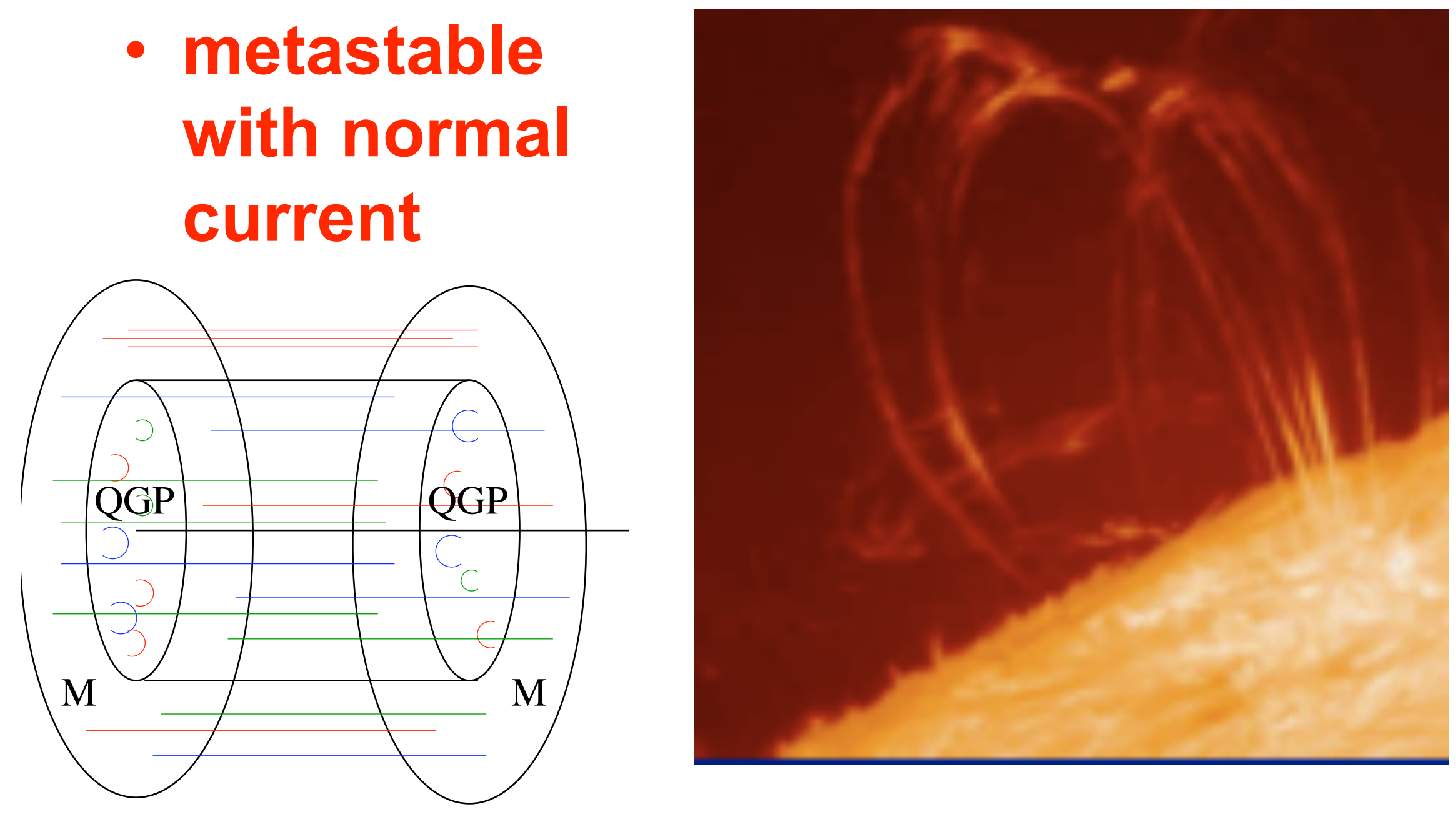


\section{Summary on monopoles}

- as T decreases, electric coupling grows and magnetic decreases $\cdot e / m$ equilibrium is around $1.4 \mathrm{Tc}$

Monopole density peaks at Tc where their mass $<300 \mathrm{MeV}$ (While $\mathrm{q}, \mathrm{g}$ and dyons are all much heavier, around $4 \mathrm{Tc}=800 \mathrm{MeV}$ )

-Correlators are very similar to that in strongly coupled Coulombic liquids: must be real objects

-Scattering between $\mathrm{e}$ - and $\mathrm{m}$-charges is large and has backward peak. Classical caging $=>$ small viscosity and diffusion

Quantum $\mathrm{g}+\mathrm{m}$ scattering produces reasonable viscosity eta/s for RHIC and predicts eta/s $=.2$ at LHC

-Monopoles behave as Bose-condenced atoms ( $\mathrm{He} 4)$ at Tc $<=$ Feynman universality and equality of clusters: must be real

objects 


\section{Summary on jet quenching, the "cone" and the two "ridges"}

- tomography $=>$ jet quenching is enhanced at Tc

- A long-time stability puzzle of the cone and two "ridges" (with and without the jet)

- dilemma: metastable flux tubes or "acoustic solution" (the second wave due to rapid change of $\left.c_{s}(t)\right)$

- Why does it all happen? => plasma is magnetic near Tc $=>$ dual MHD 